\title{
Diminished adherence of Biomphalaria glabrata embryonic cell line to sporocysts of Schistosoma mansoni following programmed knockout of the allograft inflammatory factor
}

Fernanda Sales Coelho ${ }^{1}$, Rutchanee Rodpai ${ }^{2,3}$, André Miller ${ }^{4}$, Shannon E. Karinshak²,5 ${ }^{2}$ Victoria H. Mannn ${ }^{2,5}$, Omar dos Santos Carvalho ${ }^{1}$, Roberta Lima Caldeira ${ }^{1}$, Marina de Moraes Mourão ${ }^{{ }^{*}}$, Paul J. Brindley ${ }^{2,5^{*}}$ and Wannaporn Ittiprasert ${ }^{2,5^{*}}$ (1)

\begin{abstract}
Background: Larval development in an intermediate host gastropod snail of the genus Biomphalaria is an obligatory component of the life-cycle of Schistosoma mansoni. Understanding of the mechanism(s) of host defense may hasten the development of tools that block transmission of schistosomiasis. The allograft inflammatory factor 1, AlF, which is evolutionarily conserved and expressed in phagocytes, is a marker of macrophage activation in both mammals and invertebrates. AlF enhances cell proliferation and migration. The embryonic cell line, termed Bge, from Biomphalaria glabrata is a versatile resource for investigation of the snail-schistosome relationship since Bge exhibits a hemocytelike phenotype. Hemocytes perform central roles in innate and cellular immunity in gastropods and in some cases can kill the parasite. However, the Bge cells do not kill the parasite in vitro.
\end{abstract}

Methods: Bge cells were transfected by electroporation with plasmid pCas-BgAIFx4, encoding the Cas9 nuclease and a guide RNA specific for exon 4 of the B. glabrata AIF (BgAlF) gene. Transcript levels for Cas9 and for BgAlF were monitored by reverse-transcription-PCR and, in parallel, adhesion of gene-edited Bge cells during co-culture with of schistosome sporocysts was assessed.

Results: Gene knockout manipulation induced gene-disrupting indels, frequently 1-2 bp insertions and/or 8-30 bp deletions, at the programmed target site; a range from 9 to 17\% of the copies of the $\mathrm{BgAlF}$ gene in the Bge population of cells were mutated. Transcript levels for BgAlF were reduced by up to $73 \%(49.5 \pm 20.2 \% \mathrm{SD}, P \leq 0.05, n=12)$. Adherence by BgAlF gene-edited ( $\triangle B$ gAIF) Bge to sporocysts diminished in comparison to wild type cells, although cell morphology did not change. Specifically, as scored by a semi-quantitative cell adherence index (CAI), fewer $\triangle B g A I F$ than control wild type cells adhered to sporocysts; control CAl, $2.66 \pm 0.10, \triangle B g A I F, 2.30 \pm 0.22(P \leq 0.01)$.

\footnotetext{
*Correspondence: marina.mourao@fiocruz.br; pbrindley@gwu.edu; wannaporni@gwu.edu

${ }^{1}$ Grupo de Pesquisa Em Helmintologia E Malacologia Médica, Instituto

René Rachou, Fundação Oswaldo Cruz, Belo Horizonte, MG, Brazil

${ }^{2}$ Department of Microbiology, Immunology and Tropical Medicine,

School of Medicine and Health Sciences, George Washington University,

Washington, D.C., USA

Full list of author information is available at the end of the article
}

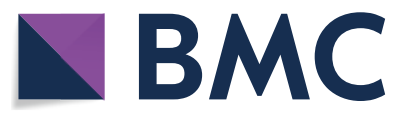

(c) The Author(s) 2020. This article is licensed under a Creative Commons Attribution 4.0 International License, which permits use, sharing, adaptation, distribution and reproduction in any medium or format, as long as you give appropriate credit to the original author(s) and the source, provide a link to the Creative Commons licence, and indicate if changes were made. The images or other third party material in this article are included in the article's Creative Commons licence, unless indicated otherwise in a credit line to the material. If material is not included in the article's Creative Commons licence and your intended use is not permitted by statutory regulation or exceeds the permitted use, you will need to obtain permission directly from the copyright holder. To view a copy of this licence, visit http://creativecommons.org/licenses/by/4.0/. The Creative Commons Public Domain Dedication waiver (http://creativecommons.org/publicdomain/zero/1.0/) applies to the data made available in this article, unless otherwise stated in a credit line to the data. 
Conclusions: The findings supported the hypothesis that BgAlF plays a role in the adherence of B. glabrata hemocytes to sporocysts during schistosome infection in vitro. This demonstration of the activity of programmed gene editing will enable functional genomics approaches using CRISPR/Cas9 to investigate additional components of the snail-schistosome host-parasite relationship.

Keywords: Biomphalaria glabrata embryonic cell line, Bge, CRISPR/Cas9, Gene editing, Allograft inflammatory factor, Cell adhesion

\section{Background}

Evolution endowed the schistosomes with a complex life-cycle that includes both a freshwater gastropod intermediate host and a definitive mammalian host. Several species of the freshwater snail genus Biomphalaria are the intermediate host for Schistosoma mansoni. The Neotropical species, Biomphalaria glabrata has been studied extensively with respect to host-parasite relationship and coevolution with $S$. mansoni, especially on mechanisms of susceptibility and/or resistance to the compatible parasites [1,2]. Genetic variation is evident among isolates and strains of B. glabrata, both in the laboratory and in the field, resulting in a spectrum of the susceptibility of infection with S. mansoni [3]. Considerable advances have been made in the exploration and characterization of mechanisms of the internal defenses system (IDS) of the snail that determine susceptibility and resistance to schistosome [4-11]. The resistance phenotype is underpinned by a complex genetic trait, where the schistosome larva fails to develop as the consequence of innate and cellular immune responses. Hemocytes of resistant snails encapsulate and destroy the sporocyst [11-18].

Biomphalaria glabrata embryonic cell line (Bge) [19] remains to date the only established cell line from any mollusk. The cell line originates from 5-day-old embryos of B. glabrata susceptible to infection with S. mansoni. The Bge cell line has been studied extensively to interrogate the host-parasite relationship because the Bge cell exhibits a hemocyte-like behavior that includes encapsulation of the larval parasite, but does not kill the parasites [20-28].

The genome sequence of $B$. glabrata has been reported [29], along with ongoing transcriptome and proteome catalogues that include factors participating in immunological surveillance, phagocytosis, cytokine responses, and pathogen recognition receptor elements including Toll-like receptors and fibrinogen-related proteins [30-36]. An orthologue of the evolutionary conserved allograft inflammatory factor (AIF) is an evolutionary conserved protein typically expressed in phagocytes and granular leukocytes in both vertebrate and invertebrate. Functions demonstrated for AIF include macrophage activation, enhancement of cellular proliferation and of migration in mammalian and invertebrate cells; protostomes and deuterostomes [37-41]. AIF also plays a key role in the protective response by $B$. glabrata to invasion by schistosomes $[8,9]$. BgAIF, the orthologue in B. glabrata is expressed in hemocytes, which participate in phagocytosis, cellular proliferation, and cellular migration. Elevated expression of $B g A I F$ is a characteristic of the resistance of $B$. glabrata to schistosome infection and has been considered as a marker of hemocyte activation $[8,9]$.

Expression of AIF is also seen during hemocyte activation in oysters [36, 38, 42, 43] and during hepatic inflammation during murine schistosomiasis [44, 45]. We hypothesized that BgAIF was involved in cell mediated immune response(s) by $B$. glabrata through activation of hemocyte cell adhesion and/or migration after the schistosome miracidium has penetrated into the tissues of the snail. We addressed this hypothesis by using CRISPR/Cas9-based programmed genome editing to interrupt the BgAIF gene of B. glabrata in the Bge cell line, following reports that indicated the utility of using CRISPR-based programmed gene knockout approach in other mollusks including the Pacific oyster, Crassostrea gigas and the slipper limpet, Crepidula fornicata and the gastropod, Lymnaea stagnalis [46-48]. As detailed below, we demonstrated the activity of programmed genome editing in Bge cells, with gene knockout at the BgAIF locus.

\section{Methods}

\section{Gene editing construct}

The gene encoding the allograft inflammatory factor of B. glabrata, BgAIF (2226 bp; accession number BGLB005061, https://www.vectorbase.org/) includes five exons interrupted by four introns (Fig. 1a). A guide RNA (gRNA) for Cas9-catalyzed gene editing specific for the target B. glabrata gene locus, BgAIF, was identified in the BGLB005061 sequence using the 'CHOPCHOP' v3 tool, https://chopchop.cbu.uib.no/, with default parameters compatible for the protospacer adjacent motif, NGG, of Cas9 from Streptococcus pyogenes [49-51] and screened for off-target sites against the B. glabrata genome [29]. Based on the guidance from the $\mathrm{CHOPCHOP}$ analysis, we chose the top ranked guide RNA (gRNA), AGA CTT 
TGT TAG GAT GAT GC, specific for exon 4 of the AIF gene, with predicted high CRISPR/Cas9 efficiency for double-stranded cleavage in tandem with an absence of off-target activity in the genome of B. glabrata (Fig. 1a). A CRISPR/Cas9 vector encoding the gRNA targeting exon 4 of BgAIF under the control of the mammalian U6 promoter and encoding Cas 9, with nuclear localization signals 1 and 2, driven by the human cytomegalovirus (CMV) immediate early enhancer and promoter was assembled using the GeneArt CRISPR nuclease vector system (Thermo Fisher Scientific, Waltham, MA, USA), according to the manufacturer's protocol. Briefly, the $20 \mathrm{nt}$ of either target (including 'GTTTT' on the $3^{\prime}$ end) or complementary to target (including 'CGGTG' on the $3^{\prime}$ end) sequences were synthesized commercially (Integrated DNA Technology, IA, USA), and annealed according to manufacturer's protocol. The annealed double-strand DNA (dsDNA) was ligated into the linearized GeneArt $^{\circledR}$ CRISPR Nuclease vector via BamHI and $B s m B I$ restriction sites, respectively, and the construct was termed pCas-BgAIFx4 (Fig. 1b). (The sequence of GeneArt CRISPR nuclease vector backbone is available at https://www.thermofisher.com/order/catalog/product/ A21174\#/A21174). Chemically competent TOP10, E. coli cells (Invitrogen, Thermo Fisher Scientific) were transformed with pCas-BgAIFx4 by the heat shock method and cultured on LB-agar supplemented with ampicillin at $100 \mu \mathrm{g} / \mathrm{ml}$. Subsequently, the integrity of the recombinant plasmids from several single colonies of ampicillin-resistant $E$. coli transformants was confirmed by amplicon PCR-based Sanger direct nucleotide sequence analysis using a U6 gene-specific primer for gRNA ligation and orientation (Fig. 1b).

\section{Biomphalaria glabrata embryonic (Bge) cell line culture}

The Bge cell line was provided by the Schistosomiasis Resource Center (SRC), Biomedical Research Institute (BRI), Rockville, MD, USA. Historically, the Bge cell line was sourced by the SRC from the American Type Culture Collection (Manassas, VA, USA), catalog no. ATCC CRL 1494 , and thereafter maintained at BRI for $>10$ years. Bge cells were maintained at $26^{\circ} \mathrm{C}$ in air in 'Bge medium, which is comprised of $22 \%$ (v/v) Schneider's Drosophila medium, $0.13 \%$ galactose, $0.45 \%$ lactalbumin hydrolysate, $0.5 \%(\mathrm{v} / \mathrm{v})$ phenol red solution, $20 \mu \mathrm{g} / \mathrm{ml}$ gentamycin, and supplemented with $10 \%$ heat-inactivated fetal bovine serum $[24,52]$. Bge cells were grown to $80 \%$ confluence before transfection by electroporation with

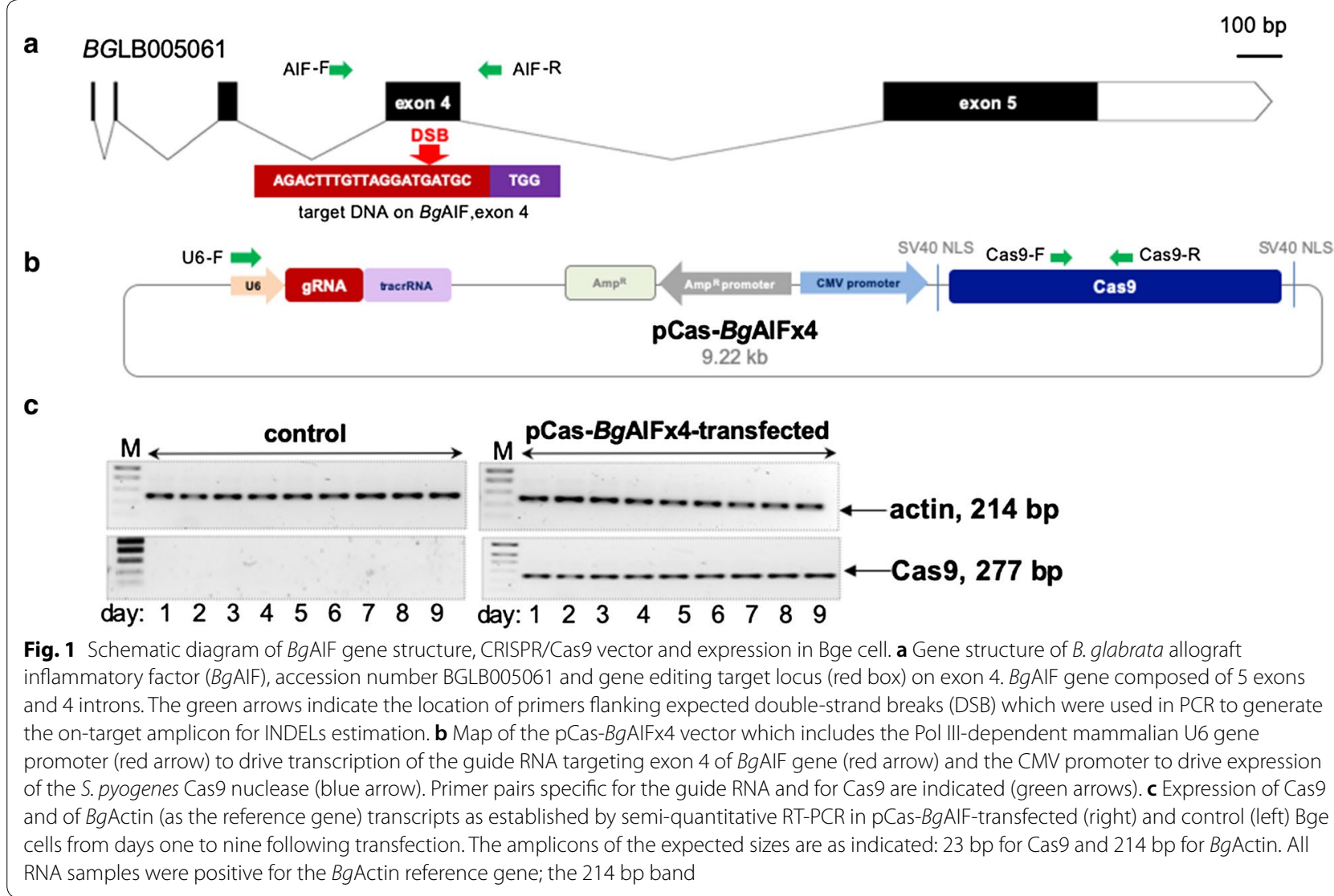


pCas-BgAIFx4. The Bge cells were free of contamination with Mycoplasma, as established with a PCR-based test (LookOut ${ }^{\circledR}$ Mycoplasma PCR Detection Kit, SigmaAldrich, St. Louis, MO, USA).

\section{Transfection of Bge cells by square wave electroporation}

Bge cells were harvested using a cell scraper, washed twice in Bge medium, counted, and resuspended at 20,000 cell/ $\mu \mathrm{l}$ in Opti-MEM medium (Sigma-Aldrich). Two million cells were transferred into $0.2 \mathrm{~mm}$ path length electroporation cuvettes (BTX Harvard Apparatus, Hollister, MA, USA) containing $6 \mu \mathrm{g}$ pCas-BgAIFx4 in $\sim 100 \mu \mathrm{l}$ Opti-MEM. The cells were subjected to electroporation using one pulse at $125 \mathrm{~V}$ for $20 \mathrm{~ms}$, using a square wave pulse generator (ECM 830, BTX Harvard Apparatus). Immediately thereafter, the Bge cells were maintained in 12-well plates (Greiner Bio-One, NC, USA) at $26{ }^{\circ} \mathrm{C}$. The mock control included Opti-MEM only for electroporation. The presence of transcripts encoding the B. glabrata actin and the Cas9 was monitored daily for 9 days following transfection by electroporation (Fig. 1c).

\section{Sequential isolation of total RNA and genomic DNA}

To monitor the transfection of Bge cell by pCas9$B g A I F x 4$, we investigated the expression of Cas9 in Bge cells by reverse transcription PCR (RT-PCR). Both total RNA and genomic DNA were extracted sequentially from cell pellets, as described [53, 54]. In brief, each sample of total RNA sample was extracted using $\mathrm{RNAzol}^{\circledR}$ RT reagent (Molecular Research Center, Inc., Cincinnati, $\mathrm{OH}, \mathrm{USA}$ ) according to the manufacturer's protocol. Subsequently, the DNA/protein pellet retained after recovery of RNA was resuspended in DNAzol ${ }^{\circledR}$ solution (Molecular Research Center, Inc), from which total DNA was recovered. The RNAs and DNAs were dissolved in nuclease-free water and their concentration and purity established by spectrophotometry (Nanodrop 1000, Thermo Fisher Scientific).

\section{Expression of Cas9 in Bge cells}

To investigate transcription from the pCas-BgAIFx4 vector following transfection of Bge cells, levels of transcribed Cas9 were investigated by semi-quantitative RTPCR. The Cas9-specific primers were Cas9-F (5'-AGC ATC GGC CTT GAT ATC GG-3') and Cas9-R (5'-AGA AGC TGT CGT CCA CCT TG-3') (Fig. 1b). Total RNA from the non-transfected $m$ ock (Opti-MEM electroporated-), and pCas-BgAIFx4 DNA electroporated-Bge cells were treated with DNase I (Ambion, Thermo Fisher Scientific) to digest any residual vector pCas-BgAIFx4
DNA and contaminating genomic DNAs. The RNAs were reverse transcribed to cDNA using ProtoScript II reverse transcriptase with oligo $\mathrm{dT}$ and random primers (First Strand cDNA Synthesis Kit, New England Biolabs, Ipswich, MA, USA). RT-PCRs specific for the Cas9 or actin gene of B. glabrata, BgActin (GenBank: U53348.1) were undertaken, with $B g$ Actin serving as the positive control for RNA integrity. The primer pairs used for the BgActin coding sequences were termed actin-F (5'-AAG CGA CGT TTT CTT GGT GC-3') and actin-R (5'-ACC CAT ACC AAC CAT CAC ACC-3'). Amplicons and molecular size standards were separated by electrophoresis through Tris-acetate-EDTA-buffered agarose $1 \%$ stained with ethidium bromide (Fig. 1c).

\section{Analysis of programmed mutation of the allograft inflammatory factor gene of $B$. glabrata}

Genomic DNA samples from the mock-transfected and pCas-BgAIFx4-transfected cells were amplified by PCR using the primers AIF-F (5'-GCA GAT TTG CAA TTC AAC ACT TA-3') and AIF-R (5'-TGC CAG CTA GCT TAC TGC AT-3') that flank the CRISPR/Cas9 programmed double-stranded break (DSB) site (Fig. 1a). Amplicons of $568 \mathrm{nt}$ in length (from residues 489 to 1056 of the BgAIF_BGLB0055061 gene) were obtained using the AIF-F and - R primer pair. Amplicons were isolated from the agarose gel using the PCR cleanup and gel extraction kit (Takara Bio, Mountain View, CA, USA) and the nucleotide sequence of amplicons determined by Sanger direct sequencing (GENEWIZ, South Plainfield, NJ, USA). Chromatograms of the sequence reads from the control and experimental groups in each replicate experiment were subjected to online analysis using the TIDE algorithm, https://tide.deskgen.com/ $[55,56]$ and also using the Inference of CRISPR v2 Edits analysis (ICE) software, https://ice.synthego.com/\#/ (Synthego Corporation, Redwood City, CA, USA) [57]. Estimates of CRISPR efficiency, insertion-deletion (INDEL)-substitution percentages, and the nucleotide sequence of mutant alleles were obtained using both the TIDE and the ICE platforms [55, 56] (Fig. 2a, b).

\section{Quantitative real time PCR analysis of transcription of BgAlF}

To evaluate the differential levels of the BgAIF transcript among the control and experimental groups, total RNAs were extracted and treated with DNase I, as above. DNase I treated-RNA (200 ng) was reverse transcribed to cDNA, followed by quantitative RT-PCR, using the ViiA7 real time PCR system (Applied Biosystems, MA, USA), and the SSoAdvanced Universal SYBR Green Supermix reagents (Bio-Rad, CA, USA), according to the manufacturer's recommendations. The following nucleotide 
primers used BgAIF gene-specific primers amplify 119257 nt of BgAIF GenBank accession number EX001601.1: BgAIF-rt-F (5'-CCT GCT TTT AAC CCG ACA GA-3') and BgAIF-rt-R (5'-TGA ATG AAA GCT CCT CGT CA-3'). Differential BgAIF gene expression was calculated after normalizing with BgActin (primers as above) and comparison with the non-treated (control) cells. The $\Delta \Delta \mathrm{Ct}$ method was used to calculate the differential gene expression [58], with assistance of the GraphPad Prism 8 software (San Diego, CA, USA) (Fig. 2c).

\section{Schistosome sporocysts}

Miracidia of the NMRI strain of S. mansoni were hatched from eggs recovered from livers of schistosome infected mice (SRC, Biomedical Research Institute, Rockville, MD, USA) under axenic conditions [28], primary sporocysts were transformed from the miracidia in vitro, as previously described [26]. Briefly, miracidia were immobilized by chilling on ice for $25 \mathrm{~min}$, followed by pelleting using centrifugation, $500 \times g$ at $4{ }^{\circ} \mathrm{C}, 60 \mathrm{~s}$. The miracidia were washed with ice cold Chernin's balanced salt solution $\left(28 \mathrm{mM} \mathrm{NaCl}, 0.5 \mathrm{mM} \mathrm{Na} 2 \mathrm{HPO}_{4}, 2 \mathrm{mM} \mathrm{KCl}\right.$, $1.8 \mathrm{mM} \mathrm{MgSO}_{4} \cdot 7 \mathrm{H}_{2} \mathrm{O}, 0.6 \mathrm{mM} \mathrm{NaHCO}$ and $3.6 \mathrm{mM}$ $\mathrm{CaCl}_{2} \cdot 2 \mathrm{H}_{2} \mathrm{O}$ ) with $1 \mathrm{mg} / \mathrm{ml}$ glucose, trehalose, and antibiotic, $10 \mu \mathrm{l} / \mathrm{ml}$ of $100 \times$ penicillin/streptomycin (Thermo Fisher Scientific), termed $\mathrm{CBSS}^{+}$. Approximately 5000 miracidia per well of a 24-well plate were cultured in $\mathrm{CBSS}+$ at $26^{\circ} \mathrm{C}$ for $24 \mathrm{~h}$, after which the sporocysts were washed to remove shed ciliated epidermal plates and other debris, followed by transfer to a $1.5 \mathrm{ml}$ microcentrifuge tube [26].
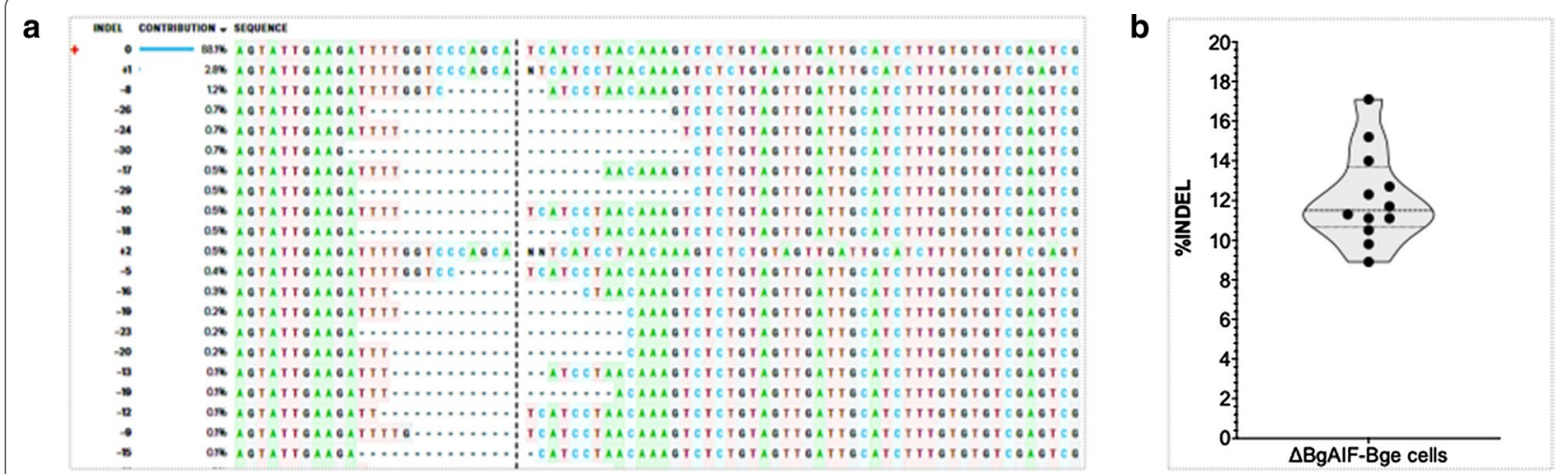

c

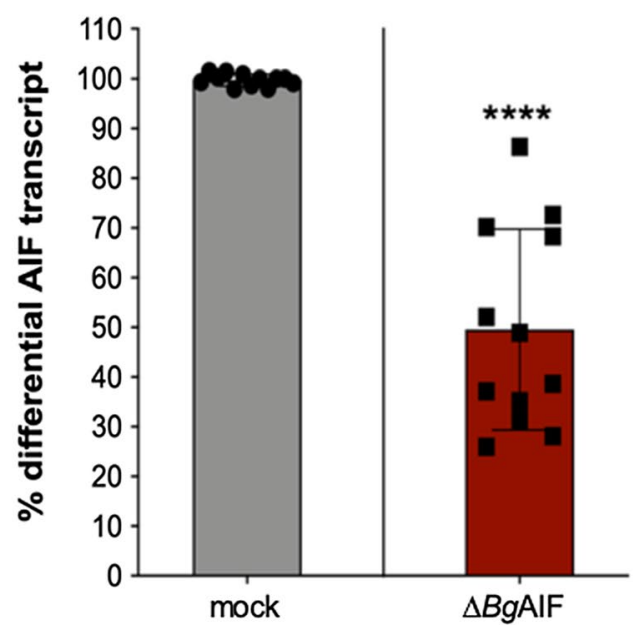

Fig. 2 Establishment of BgAlF-knockout lines of Bge cells. a Representative examples of frequent gene insertions-deletions (1-2 bp insertions and 8-30 bp deletions, straddling the programmed CRISPR/Cas9-induced double-stranded break in exon 4, as determined by ICE software-based analysis. b TIDE algorithm-based violin plot of insertion-deletion percentages (\% INDEL) computed using the amplicon sequence traces from the 12 biological replicates of pCas-BgAlF-transfected Bge cell populations. c Reduction of BgAlF transcription by about 50\% following programmed genome editing of Bge cells ( $\triangle B$ gAlF-Bge) in comparison to control Bge cells. Mean transcript reduction, $49.55 \pm 20.22 \%(S D), P \leq 0.0001^{* * * *}(n=12)$ (unpaired Student's t-test) 


\section{Sporocyst-Bge cell binding assay and cell adhesion index} (CAI)

To investigate the if BgAIF would affect the ability of cell adhesion to S. mansoni sporocysts, we co-cultured the non-transfected Bge cell or non-selected-, transfected-pCas-BgAIFx4 cells (BgAIF depleted-cells named ' $\triangle B g$ AIF-Bge') with in vitro transformed sporocysts, then the cell adhesion index (CAI) was calculated as previously described [26, 59]. A limitation of this study was that we were not be able to select or enrich for BgAIF edited-cells, and hence the $\Delta B g$ AIF-Bge cell populations can be considered to be a population of gene mutated mixed with non-modified (wild type) cells. CAI is a semiquantitative method of cell adhesion to primary sporocysts using four categories of scores ranging from one to four-lower to higher numbers of cells adherent to the parasite's surface. In brief, we mixed single cell suspensions of 500,000 Bge cells with 200 freshly-prepared sporocysts (total volume $200 \mu \mathrm{l}$ of $\mathrm{CBSS}^{+}$) in sterile, siliconized tubes (Bio Plas, Thomas Scientific, Swedesboro, NJ, USA). The Bge cell-sporocyst co-culture was maintained at $26^{\circ} \mathrm{C}$ for $24 \mathrm{~h}$. Cellular morphology and adhesion of the cells to the surface of the sporocysts was monitored and recorded using an inverted microscope, at $20 \times$ magnification (Zeiss Axio Observer A1, Carl Zeiss LLC, White Plains, NY, USA) after gently transferring the parasite-cell suspension to a tissue culture plate (Greiner Bio-One). Scoring of the adherence index was carried out in a blinded fashion to the investigator reading the score; $\geq 50$ sporocysts from each experimental group were counted each time, and triplicates of each treatment group were scored. Seven independent biological replicates of this CAI-based sporocyst-Bge cell binding assay were carried out. In total, $\geq 400$ sporocysts were examined from each treatment and control group. Averages for the CAI values were calculated from the cell adhesion scores ranging from 1 to 4 (examples presented in Fig. 3a) according to the formula, $\mathrm{CAI}=$ total binding value per number of sporocysts [26].

\section{Results}

\section{Cas9 nuclease transcribed in transfected Bge cells}

Total RNA was extracted from non-transfected cell (wild type; WT), mock control and pCas-BgAIFx4-transfected Bge cells to assess the expression of Cas9 (Fig. 1b). The cDNAs from either controls or pCas-BgAIFx4-transfected Bge cells were employed as the template in PCRs using two primer pairs, one specific for Cas9 and the other for BgActin, the actin gene of B. glabrata that served as the reference gene (Fig. 1b, c). Transcripts encoding Cas9 in transient pCas-BgAIFx 4 transfectedBge cells were detected at $24 \mathrm{~h}$ after transfection and expression was maintained for the 9 days of the assay.
The specific amplicon of Cas9 mRNA (277 bp) was observed from pCas-BgAIFx4 transfected cells, but was absent from the non-transfected cells (Fig. 1c). Our findings supported previous findings that revealed a CMV promoter driven by luciferase in Bge cells [60]. Expression of the control reference BgActin was observed at a $214 \mathrm{bp}$ amplicon in both the controls and experimental samples (Fig. 1c).

\section{Programmed mutation of BgAlF confirmed functional CRISPR/Cas9 activity in Bge cells}

Genomic DNAs from wild type Bge, medium-transfected (mock) and pCas-BgAIFx4-transfected cells were used as the template for PCRs with the primer pair, AIF-F and AIF-R, flanking the programmed Cas9 cleavage site on BgAIF, exon 4 (Fig. 1a, green arrows; amplicon size, $\sim 200$ nt). The red arrow indicates the predicted site of the Cas9-catalyzed double-strand break (DSB) within the BgAIF locus (Fig. 1a). The nucleotide sequence of the amplicons was determined by Sanger direct sequencing using the same primers. Both forward and reverse Sanger direct sequencing reads from the same amplicon were estimated for insertion-deletion (INDELs) by the ICE and the TIDE algorithms [55, 56]. The reads from the Bge cells transfected with the pCas9-BgAIFx4 contained INDELs at or around the programmed CRISPR/ Cas9 cleavage site. The percentage of reads that included INDELs ranged from $8.9 \%$ to $17.1 \%$, in the 12 biological replicates that were carried out (Fig. 2a, b). Notably, the mutation profile in the vicinity of the predicted DSB in BgAIF was similar among these 12 replicates, which were undertaken independently. Commonly observed INDELs at the DSBs site as revealed by the ICE analysis included deletions of 8 to $30 \mathrm{bp}$ and insertions of 1 or 2 bp (Fig. 2a). These mutations were predicted to result in frameshift mutations, the consequent loss of the open reading frame, and hence and permanent knockout of $B g$ AIF in the gene-edited Bge cell. The profile of the frequency of mutations observed in each biological replicate was used to plot the curve (Prism 8 software; https ://www.graphpad.com/) presented in Fig. 2b. These findings demonstrated that programmed genome editing using CRISPR/Cas9 was active in Bge cells, and that the non-homologous end-joining (NHEJ) pathway [61] was active in B. glabrata for the repair of programmed double-stranded breaks, leading to targeted gene knockout.

\section{Programmed mutation interrupted expression of BgAlF}

The aims of the study included the investigation of the activity of CRISPR/Cas9 gene editing in the Bge cell line and addressing the hypothesis that AIF functions in the activation of a macrophage like phenotype by the Bge cell. Accordingly, Bge cells were transfected 


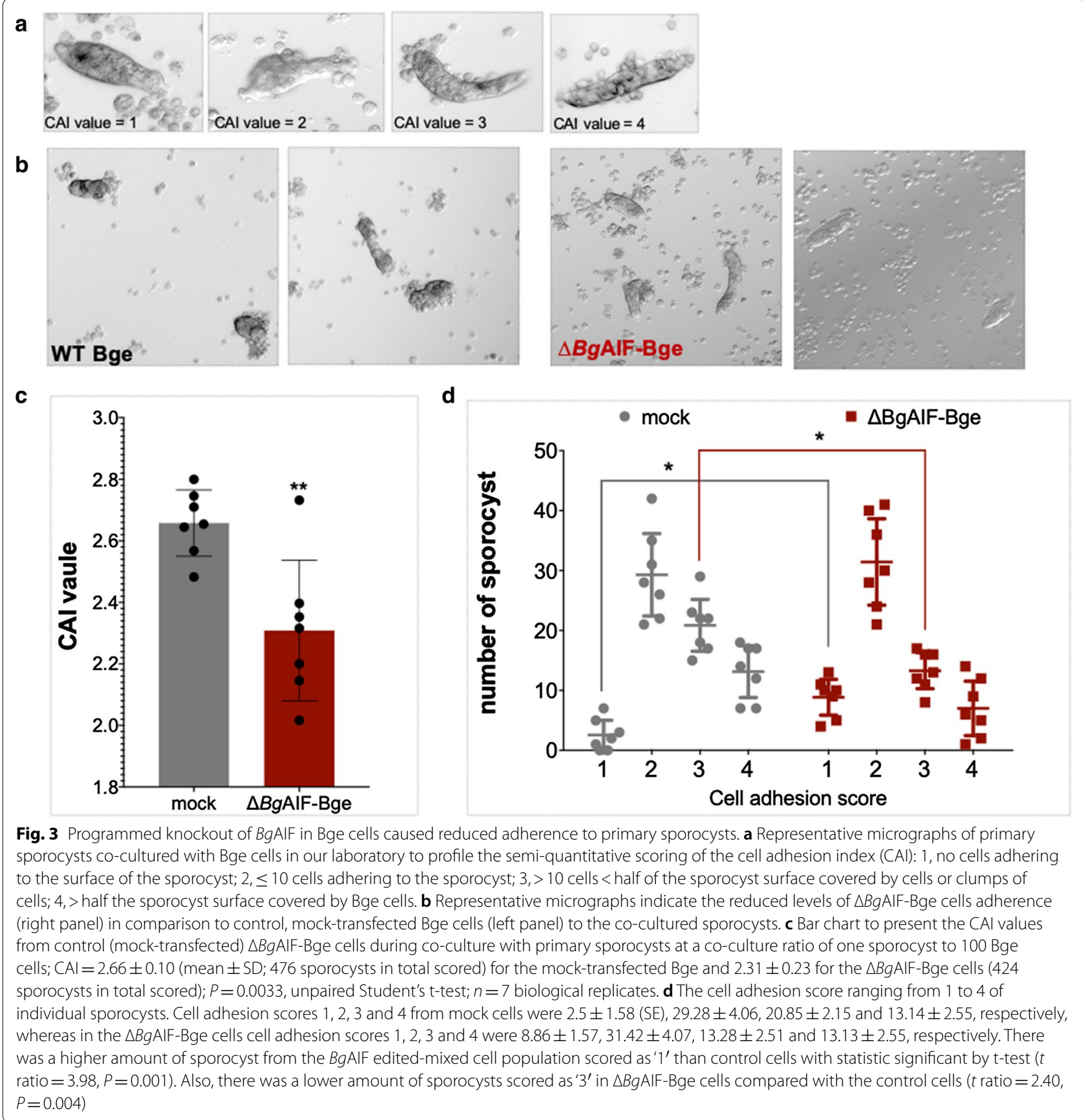

with pCas9-BgAIFx4 plasmid DNA. The experimental approach did not include drug resistance and/or reporter gene markers in order to enrich for transfected Bge cells. However, even without enrichment for transfected cells, there was a highly statistically significant reduction in levels of BgAIF transcripts in the transfected Bge cell population. Expression of BgAIF transcripts were assessed using RNAs from the cells at 9 days post-transfection. Comparison of the experimental and control groups revealed significantly reduced levels of the $B g A I F$ in the pCas-BgAIFx4-transfected cells, mean $49.55 \pm 20.22 \%$, range 28.1 to $86.3 \%(n=12)$ compared to the wild type Bge (normalized sample, $100 \%$ expression), mock control cells (unpaired t-test and F-test to compare variances; $F_{(11,11)}=294.7, P<0.0001$ ) (Fig. 1b). An inverse correlation between the percentage of INDELs and reduction in transcript levels was not apparent (not shown). 
Programmed knockout of BgAIF interfered with adherence of Bge cells to schistosome sporocysts

Single-cell suspensions of Bge cells in the mock-treated and $\triangle B g$ AIF groups were co-cultured for $24 \mathrm{~h}$ in siliconized tubes with primary S. mansoni sporocysts. At that point, the numbers of cells that had adhered to each sporocyst were scored. This was accomplished by examination of at least 5 discrete sites of the well of the 24-well plate with $\geq 50$ sporocysts of each group. The cell adhesion index (CAI) were scored from 1 to 4 , with a score of 1 indicating few or no adherent cells and a score of 4 indicating that cells or clumps of cells covered more than half the tegumental surface of the sporocyst, as defined in earlier reports [26] (Fig. 3a). Cells from the mocktransfected control group mostly adhered in clumps or singly to the surface of the parasite (representative images in the upper panels of Fig. 3b), with CAI values that ranged from 2 to 4 . By contrast, fewer cells adhered to the surface of the sporocysts in the $\Delta B g$ AIF-Bge group (representative images, lower panels in Fig. 3b), with CAI values ranging from 2 to 3 . Only $\sim 20 \%$ of the $\triangle B g$ AIFBge cells adhered to the surface of the sporocysts and most of the cells retained remained spread singly on the surface of the well of tissue culture plate (Fig. 3b). The CAI values ascertained from the seven biological replicates ( $>50$ parasites in each replicate) with more than 400 parasites in total were scored). The average CAI were $2.66 \pm 0.10$ (range: $2.53-2.78$ ) and $2.25 \pm 0.22$ (range, 2.08 to 2.55 ) in the mock-treated and transfection control group, respectively. The average of CAI value from control group was significantly higher than the $\triangle B g$ AIF group (Fig. 3c) (unpaired t-test: $t=3.661$ $d f=12, P=0.0033)$. More specifically, the CAI categoryspecific CAI values for mock-treated cells averaged from the cell adherence to single sporocysts, were $2.5 \pm 1.58$ (SE), $29.28 \pm 4.06,20.85 \pm 2.15$, and $13.14 \pm 2.55$ for categories 1, 2, 3 and 4, respectively. For the $\triangle B g$ AIFBge cells, the CAI values were $8.86 \pm 1.57,31.42 \pm 4.07$, $13.28 \pm 2.51$, and $13.13 \pm 2.55$ for categories $1,2,3$ and 4 , respectively. Although we observed CAI scores of 1 to 4 in both mock control cells and $\triangle B g$ AIF cells, nonetheless there were statistically significant higher numbers of sporocysts with the lowest adherence, scored as ' 1 ', in the $\triangle B g$ AIF group compared with the mock control group as confirmed using a multiple t-test $(t=3.98$, $d f=12, P=0.001$ ). By contrast, there were significantly higher numbers of sporocysts scored as ' 3 ' in the control compared with the $\Delta B g$ AIF group $(t=3.52032, d f=12$, $P=0.004)$ (Fig. $3 \mathrm{~d}$ ). Finally, morphological changes were not apparent between the $\Delta B g$ AIF and the control group Bge cells.

\section{Discussion}

This report describes a novel use of programmed genome editing by the CRISPR/Cas9 approach in the embryonic cell line from the $g$ astropod snail, B. glabrata, an intermediate host snail of the human blood fluke, S. mansoni. The Bge cell line is an informative tool in investigation of snail-schistosome, host-parasite interactions. A key attribute of the Bge cell is its hemocyte-like phenotype, given the central role of the snail hemocyte in innate and cellular immunity. However, even though Bge cells adhere to the schistosome, the parasite is not killed by these cells in vitro. The allograft inflammatory factor 1 (AIF) is a conserved calcium-binding protein typically expressed in phagocytic and granular leukocytes and is a marker of macrophage activation [38, 41, 45, 62-65]. An orthologue, termed $B g$ AIF, is highly expressed in isolates of $B$. glabrata that are resistant to infection with $S$. mansoni and this gene may be linked to hemocyte activation $[8,9]$. Here, we targeted the AIF gene of B. glabrata embryonic cell line using programmed gene knockout to further interrogate its role in the intermediate host- schistosome interaction. We constructed a plasmid vector encoding the CRISPR/Cas9 nuclease and a guide RNA targeting exon 4 of BgAIF gene and the Cas9 nuclease from Streptococcus pyogenes. Bge cells were transfected with the gene-editing construct by square wave electroporation. Transcript levels of BgAIF were significantly reduced by up to $71.9 \%$ following transformation. In parallel, sequence reads of amplicons spanning the locus targeted for programmed gene knock-out revealed on-target mutation on the BgAIF gene, that had been repaired by non-homologous end joining leading to gene-inactivating insertions and deletions. In addition, the adherence of gene-edited Bge cells to sporocysts was significantly impeded in comparison to control cells, as ascertained using a semi-quantitative, cell adherence index. In our study, the \% INDELs (8.9-17.1\%) resulting from NHEJ after CRISPR/Cas9 gene editing on BgAIF exon 4 locus did not correlate with its transcript reduction $(\sim 50 \%)$ in all experimental samples. Nonetheless, alternative mechanisms could be used as the fusion of suppressors with a 'dead' Cas9 which enables gene regulation and increase the level of repression of the target gene [66].

The B. glabrata IDS comprises hemocytes and soluble proteins found in the hemolymph, among them the BgAIF [67-69]. The response of resistant mollusks is given by the adherence and encapsulation of sporocysts by hemocytes, leading to the parasite destruction [70]. The AIF-1 was demonstrated to be a pro-inflammatory cytokine that regulates immune-related genes of the oyster, Crassostrea ariakensis [36]. An orthologue in the leech Hirudo medicinalis promotes macrophagelike migration by a chemotactic activity, in addition to 
being involved in the innate immune responses as also seen in other species [41]. The adherence of the mixed populations of BgAIF gene-edited/non-edited-Bge cells to sporocysts was significantly impeded in comparison to control cells, as ascertained using a semi-quantitative cell adherence index. These cells, albeit in a low percentage, are less responsive to the $S$. mansoni parasite. These data suggested that, in the presence of S. mansoni, the Bge cells need to secrete BgAIF for activating the recruitment of more adherent Bge cells. Thus, the BgAIF protein appears to play a role in cell recognition, migration, and/or adhesion, and to participate in the early immune response to the parasite. The AIF gene is conserved broadly among protostomes and deuterostomes, including vertebrates, and also in prebilaterian including sponges, where it likely performs similar functional roles in macrophage activation and migration [71]. In humans, the HmAIF1 is an NF- $\mathrm{KB}$ pathway regulator, a pathway that comprises a family of evolutionarily conserved proteins, important to the immune system by participating in the expression of other proteins related to the immune system [72, 73]. Although more studies will be required to decipher the regulation of these pathways in B. glabrata, after the pathogen invasion, the BgAIF possibly acts throughout the activation of the NF-kB pathway, leading to the recruitment of hemocytes and consequent pathogen elimination [72, 74].

These findings confirmed the tractability of transfection of Bge cells by electroporation with the genomeediting construct, pCas-BgAIFx4, and that the CMV promoter drove transcription of Cas9 in this snail species. Whereas transformation by plasmid DNA of Bge cells by square wave electroporation appears to be novel, Bge cells have been transformed using DNA complexed with cationic lipid-based transfection reagents and with polyethyleneimine [23]. Nevertheless, our study has some limitations. Thus far we have yet to enrich the transfected cells from wild type cells. Future studies using a drug selectable marker can be designed to address this issue. Other approaches to deliver the CRISPR/Cas geneediting cargo can be tried including repeated inoculation with ribonuclear protein complexes [75], titration of the transfection chemicals [76], titration of electroporation parameters [77], and/or transduction by lentiviral virions encoding the gRNA and S. pyogenes Cas9 nuclease as we have demonstrated with eggs of S. mansoni $[53,54]$. Moreover, CIRCLE-Seq and similar approaches can be employed to investigate the off-target mutations [78].

\section{Conclusions}

Here we demonstrated CRISPR/Cas-based gene editing in a cell line from a medically important taxon of freshwater gastropods that are vectors for the transmission of schistosomiasis. We showed the functional role of a $B$. glabrata allograft inflammatory factor in the recognition/ attachment of $S$. mansoni sporocysts in vitro. The demonstration of the activity of CRISPR/Cas9 gene editing in Bge cells suggests that genome editing in the germline and somatic tissues of intact B. glabrata snails will also be functional. Whereas improvements can be anticipated in these approaches, an obvious next step will be to gene edit the intact snail B. glabrata. Transfection of germline cells within the snail using microinjection can be considered [79]. These findings, together with the first application of the CRISPR/Cas technique in the genetic edition of the snail Lymnaea stagnalis [48] are a step-change since they can favor the creation of a genetically modified Biomphalaria line to study the biology and physiology of the snail as well the schistosome-intermediate host relationship. Functional genomics using CRISPR/Cas-based genome editing in schistosomes and other trematodes responsible for major neglected tropical diseases has been reported $[53,54]$. The establishment of a functional genomic protocols involving programmed gene editing to address fundamental questions in this host-parasite relationship using genetically modified snails and schistosomes now seems to be feasible.

\begin{abstract}
Abbreviations
AlF: Allograft inflammatory factor 1; Bge: Biomphalaria glabrata Embryonic cell line; Cas9: CRISPR associated protein 9; RNA: Ribonucleic acid; PCR: Polymerase chain reaction; CAl: Cell adherence index; CRISPR: Clustered regularly-interspaced short palindromic repeats; gRNA: Guide RNA; CMV: Cytomegalovirus; DBS: Double-strand break; SRC: Schistosomiasis Resource Center; BRI: Biomedical Research Institute; RT-PCR: Reverse transcription PCR; DNA: Deoxyribonucleic acid; CDNA: Complementary deoxyribonucleic acid; IDS: Internal defense system; EDTA: Ethylenediaminetetraacetic acid; INDEL: Insertion-deletion mutation; TIDE: Tracking of indels by decomposition; ICE: Inference of CRISPR edits analysis; DNase I: Deoxyribonuclease I; CBSS: Chernin's balanced salt solution; WT: Wild type; NHEJ: Non-homologous end-joining; NF-KB: Nuclear factor kappa-B.
\end{abstract}

\section{Acknowledgements}

We thank Dr. Mathilde Knight for informative discussions on the snail-schistosome immunobiology and Dr. Margaret Mentink-Kane for support with the parasites. Bge cells were provided by the NIAID Schistosomiasis Resource Center of the Biomedical Research Institute, Rockville, Maryland through $\mathrm{NIH}-$ NIAID contract HHSN272201000005I for distribution through BEl Resources.

\section{Authors' contributions}

WI, MM and PJB designed the study. FSC and WI wrote the protocol. MMM VMH and PJB reviewed the protocol. WI served as study team leader and director. VHM and AM obtained ethical approval for vertebrate animal use. FSC, RR, AM and SEK conducted the gene editing and cell culture experiments. FSC, RR and WI performed gene mutation analysis. FSC, OSC and RCL performed CAI analysis. WI, MMM and PJB wrote the manuscript. All authors read and approved the final manuscript.

\section{Funding}

This work was supported by the Coordenação de Aperfeiçoamento de Pessoal de Nível Superior (CAPES) - finance code 001, Productivity fellowship from Conselho Nacional de Desenvolvimento Científico e Tecnológico (CNPq) granted to MMM (302518/2018-5) and RLQ (308869/2017-6) and Fapemig (APQ-01766-15), Brazil. This research was supported in part by the Wellcome 
Trust (strategic award number 107475/Z/15/Z, Functional Genomics Flatworms Initiative, K. H. Hoffmann, principal investigator, PJB, co-investigator). For the purpose of Open Access, the authors have applied a CC BY public copyright license to any Author Accepted Manuscript version arising from this submission.

\section{Availability of data and materials}

Data supporting the conclusions of this article are included within the article. The raw datasets used and analyzed during the present study are available from the corresponding authors upon reasonable request.

\section{Ethics approval and consent to participate}

The protocols and procedures to maintain S. mansoni life-cycle in mice performed at the NIAID Schistosomiasis Resource Center of the Biomedical Research Institute, Rockville, MD, USA, were approved by the Institutional Animal Care and Use Committee (IACUC) (protocol number 18-04) and followed by United States Animal Welfare Act and George Washington University IACUC policies (assurance number A3205-01).

\section{Consent for publication}

Not applicable.

\section{Competing interests}

The authors declare that they have no competing interests.

\section{Author details}

${ }^{1}$ Grupo de Pesquisa Em Helmintologia E Malacologia Médica, Instituto René Rachou, Fundação Oswaldo Cruz, Belo Horizonte, MG, Brazil. ${ }^{2}$ Department of Microbiology, Immunology and Tropical Medicine, School of Medicine and Health Sciences, George Washington University, Washington, D.C., USA. ${ }^{3}$ Department of Parasitology, Faculty of Medicine, Khon Kaen University, Khon Kaen province, Thailand. ${ }^{4}$ Schistosomiasis Resource Center, Biomedical Research Institute, Rockville, MD, USA. ${ }^{5}$ Research Center for Neglected Diseases of Poverty, School of Medicine and Health Sciences, George Washington University, Washington, D.C., USA.

Received: 3 August 2020 Accepted: 30 September 2020

Published online: 13 October 2020

\section{References}

1. Webster JP, Davies CM. Coevolution and compatibility in the snail-schistosome system. Parasitology. 2001;123(Suppl):S41-56.

2. El-Ansary A, Al-Daihan S. Important aspects of Biomphalaria snail-schistosome interactions as targets for antischistosome drug. Med Sci Monit. 2006;12:RA282-RA292.

3. Richards CS, Shade PC. The genetic variation of compatibility in Biomphalaria glabrata and Schistosoma mansoni. J Parasitol. 1987;73:1146-51.

4. Knight M, Miller A, Liu Y, Scaria P, Woodle M, Ittiprasert W. Polyethyleneimine (PEI) mediated siRNA gene silencing in the Schistosoma mansoni snail host Biomphalaria glabrata. PLoS Negl Trop Dis. 2011:5:e1212.

5. Jannotti-Passos LK, Andrade HM, Caldeira RL, Romanha AJ, Murta SM, Chapeaurouge DA, et al. Proteome analysis of the cardiac and pericardial tissue of Biomphalaria tenagophila populations susceptible and resistant to Schistosoma mansoni infection. Acta Trop. 2008;105:229-34.

6. Hanington PC, Lun CM, Adema CM, Loker ES. Time series analysis of the transcriptional responses of Biomphalaria glabrata throughout the course of intramolluscan development of Schistosoma mansoni and Echinostoma paraensei. Int J Parasitol. 2010;40:819-31.

7. Pila EA, Sullivan JT, Wu XZ, Fang J, Rudko SP, Gordy MA, et al. Haematopoiesis in molluscs: a review of haemocyte development and function in gastropods, cephalopods and bivalves. Dev Comp Immunol. 2016;58:119-28.

8. Lockyer AE, Emery AM, Kane RA, Walker AJ, Mayer CD, Mitta G, et al. Early differential gene expression in haemocytes from resistant and susceptible Biomphalaria glabrata strains in response to Schistosoma mansoni. PLoS ONE. 2012;7:e51102.

9. Larson MK, Bender RC, Bayne CJ. Resistance of Biomphalaria glabrata 13-16-R1 snails to Schistosoma mansoni PR1 is a function of haemocyte abundance and constitutive levels of specific transcripts in haemocytes. Int J Parasitol. 2014;44:343-53.

10. Pinaud S, Portela J, Duval D, Nowacki FC, Olive MA, Allienne JF, et al. A shift from cellular to humoral responses contributes to innate immune memory in the vector snail Biomphalaria glabrata. PLoS Pathog. 2016;12:e1005361.

11. Barbosa L, Caldeira RL, Carvalho OS, Vidigal TH, Jannotti-Passos LK, Coelho PM. Resistance to Schistosoma mansoni by transplantation of APO Biomphalaria tenagophila. Parasite Immunol. 2006;28:209-12.

12. Adema CM, Loker ES. Digenean-gastropod host associations inform on aspects of specific immunity in snails. Dev Comp Immunol. 2015;48:275-83.

13. Coustau C, Gourbal B, Duval D, Yoshino TP, Adema CM, Mitta G. Advances in gastropod immunity from the study of the interaction between the snail Biomphalaria glabrata and its parasites: a review of research progress over the last decade. Fish Shellfish Immunol. 2015;46:5-16.

14. Mitta G, Adema CM, Gourbal B, Loker ES, Theron A. Compatibility polymorphism in snail/schistosome interactions: from field to theory to molecular mechanisms. Dev Comp Immunol. 2012;37:1-8.

15. Mitta G, Gourbal B, Grunau C, Knight M, Bridger JM, Theron A. The compatibility between Biomphalaria glabrata snails and Schistosoma mansoni: an increasingly complex puzzle. Adv Parasitol. 2017;97:111-45.

16. Pila EA, Li H, Hambrook JR, Wu X, Hanington PC. Schistosomiasis from a snail's perspective: advances in snail immunity. Trends Parasitol. 2017;33:845-57.

17. Famakinde DO. Molecular context of Schistosoma mansoni transmission in the molluscan environments: a mini-review. Acta Trop. 2017;176:98-104

18. Knight M, Arican-Goktas HD, Ittiprasert W, Odoemelam EC, Miller AN, Bridger JM. Schistosomes and snails: a molecular encounter. Front Genet. 2014:5:230.

19. Hansen EL. A cell line from embryos of Biomphalaria glabrata (Pulmonata): establishment and characteristics. In: Maramorosch K, editor. Invertebrate Tissue Culture: Research Applications. Cambridge: Academic Press, Inc:; 1976. p. 75-99.

20. Laursen JR, Yoshino TP. Biomphalaria glabrata embryonic (Bge) cell line supports in vitro miracidial transformation and early larval development of the deer liver fluke Fascioloides magna. Parasitology. 1999;1 18:187-94.

21. Wheeler NJ, Dinguirard N, Marquez J, Gonzalez A, Zamanian M, Yoshino TP, et al. Correction to: sequence and structural variation in the genome of the Biomphalaria glabrata embryonic (Bge) cell line. Parasit Vectors. 2018;11:566.

22. Wheeler NJ, Dinguirard N, Marquez J, Gonzalez A, Zamanian M, Yoshino $T P$, et al. Sequence and structural variation in the genome of the Biomphalaria glabrata embryonic (Bge) cell line. Parasit Vectors. 2018;11:496.

23. Rinaldi G, Yan H, Nacif-Pimenta R, Matchimakul P, Bridger J, Mann VH, et al. Cytometric analysis, genetic manipulation and antibiotic selection of the snail embryonic cell line Bge from Biomphalaria glabrata, the intermediate host of Schistosoma mansoni. Int J Parasitol. 2015;45:527-35.

24. Odoemelam E, Raghavan N, Miller A, Bridger JM, Knight M. Revised karyotyping and gene mapping of the Biomphalaria glabrata embryonic (Bge) cell line. Int J Parasitol. 2009;39:675-81.

25. Vermeire JJ, Boyle JP, Yoshino TP. Differential gene expression and the effects of Biomphalaria glabrata embryonic (Bge) cell factors during larval Schistosoma mansoni development. Mol Biochem Parasitol. 2004;135:153-7.

26. Castillo MG, Yoshino TP. Carbohydrate inhibition of Biomphalaria glabrata embryonic (Bge) cell adhesion to primary sporocysts of Schistosoma mansoni. Parasitology. 2002;125:513-25.

27. Humphries JE, Elizondo L, Yoshino TP. Protein kinase $C$ regulation of cell spreading in the molluscan Biomphalaria glabrata embryonic (Bge) cell line. Biochim Biophys Acta. 2001;1540:243-52.

28. Yoshino TP, Laursen JR. Production of Schistosoma mansoni daughter sporocysts from mother sporocysts maintained in synxenic culture with Biomphalaria glabrata embryonic (Bge) cells. J Parasitol. 1995;8:714-22.

29. Adema CM, Hillier LW, Jones CS, Loker ES, Knight M, Minx P, et al. Whole genome analysis of a schistosomiasis-transmitting freshwater snail. Nat Commun. 2017:8:15451.

30. Dheilly NM, Duval D, Mouahid G, Emans R, Allienne JF, Galinier R, et al. A family of variable immunoglobulin and lectin domain containing 
molecules in the snail Biomphalaria glabrata. Dev Comp Immunol. 2015;48:234-43.

31. Zhang SM, Loker ES. Representation of an immune responsive gene family encoding fibrinogen-related proteins in the freshwater mollusc Biomphalaria glabrata, an intermediate host for Schistosoma mansoni. Gene. 2004;341:255-66.

32. Portet A, Pinaud S, Tetreau G, Galinier R, Cosseau C, Duval D, et al. Integrated multi-omic analyses in Biomphalaria-Schistosoma dialogue reveal the immunobiological significance of FREP-SmPoMuc interaction. Dev Comp Immunol. 2017;75:16-27.

33. Hanington PC, Zhang SM. The primary role of fibrinogen-related proteins in invertebrates is defense, not coagulation. J Innate Immun. 2011:3:17-27.

34. Adema CM. Fibrinogen-related proteins (FREPs) in mollusks. Results Probl Cell Differ. 2015;57:111-29.

35. Gordy MA, Pila EA, Hanington PC. The role of fibrinogen-related proteins in the gastropod immune response. Fish Shellfish Immunol. 2015;46:39-49.

36. XuT, Xie J, Zhu B, Liu X, Wu X. Allograft inflammatory factor 1 functions as a pro-inflammatory cytokine in the oyster Crassostrea ariakensis. PLoS ONE. 2014;9:e95859.

37. Ai XL, Yao F, Wang XJ, Duan DB, Li K, Hu ZY, et al. Role of allograft inflammatory factor- 1 in regulating the proliferation, migration and apoptosis of colorectal cancer cells. Nan Fang Yi Ke Da Xue Xue Bao. 2018;38:511-9.

38. Zhang Y, Li J, Yu F, He X, Yu Z. Allograft inflammatory factor-1 stimulates hemocyte immune activation by enhancing phagocytosis and expression of inflammatory cytokines in Crassostrea gigas. Fish Shellfish Immunol. 2013;34:1071-7.

39. Jia J, Cai Y, Wang R, Fu K, Zhao YF. Overexpression of allograft inflammatory factor-1 promotes the proliferation and migration of human endothelial cells (HUV-EC-C) probably by up-regulation of basic fibroblast growth factor. Pediatr Res. 2010;67:29-34.

40. Autieri MV, Carbone CM. Overexpression of allograft inflammatory factor-1 promotes proliferation of vascular smooth muscle cells by cell cycle deregulation. Arterioscler Thromb Vasc Biol. 2001;21:1421-6.

41. Schorn T, Drago F, Tettamanti G, Valvassori R, de Eguileor M, Vizioli J, et al. Homolog of allograft inflammatory factor-1 induces macrophage migration during innate immune response in leech. Cell Tissue Res. 2015;359:853-64.

42. Martin-Gomez L, Villalba A, Carballal MJ, Abollo E. Molecular characterisation of TNF, AIF, dermatopontin and VAMP genes of the flat oyster Ostrea edulis and analysis of their modulation by diseases. Gene. 2014;533:208-17.

43. Li J, Chen J, Zhang Y, Yu Z. Expression of allograft inflammatory factor-1 (AIF-1) in response to bacterial challenge and tissue injury in the pearl oyster Pinctada martensii. Fish Shellfish Immunol. 2013;34:365-71.

44. Chen QR, Guan F, Song SM, Jin JK, Lei DS, Chen CM, et al. Allograft inflammatory factor-1 alleviates liver disease of BALB/C mice infected with Schistosoma japonicum. Parasitol Res. 2014;113:2629-39.

45. Chen QR, Guan F, Yan DJ, Lei DS, Fu L, Xia HS, et al. The dynamic expression of allograft inflammatory factor-1 in hepatic tissues and splenic cells of BALB/c mice with Schistosoma japonicum infection. Tissue Antigens. 2012;79:33-41.

46. Yu H, Li H, Li Q, Xu R, Yue C, Du S. Targeted gene disruption in Pacific oyster based on CRISPR/Cas9 ribonucleoproteincomplexes. Mar Biotechnol (NY). 2019;21:301-9.

47. Perry KJ, Henry JQ. CRISPR/Cas9-mediated genome modification in the mollusc Crepidula fornicata. Genesis. 2015;53:237-44.

48. Abe M, Kuroda R. The development of CRISPR for a mollusc establishes the formin $L s d i a 1$ as the long-sought gene for snail dextral/sinistral coiling. Development. 2019;146:dev175976.

49. Labun K, Montague TG, Gagnon JA, Thyme SB, Valen E. CHOPCHOP V2: a web tool for the next generation of CRISPR genome engineering. Nucleic Acids Res. 2016;44:W272-W276276.

50. Labun K, Montague TG, Krause M, Torres Cleuren YN, Tjeldnes H, Valen E. CHOPCHOP v3: expanding the CRISPR web toolbox beyond genome editing. Nucleic Acids Res. 2019;47:W171-W174174.

51. Montague TG, Cruz JM, Gagnon JA, Church GM, Valen E. CHOPCHOP: a CRISPR/Cas9 and TALEN web tool for genome editing. Nucleic Acids Res. 2014;42:W401-W407407.
52. Knight $\mathrm{M}$, Ittiprasert $\mathrm{W}$, Odoemelam EC, Adema CM, Miller A, Raghavan $\mathrm{N}$, et al. Non-random organization of the Biomphalaria glabrata genome in interphase Bge cells and the spatial repositioning of activated genes in cells co-cultured with Schistosoma mansoni. Int J Parasitol. 2011;41:61-70.

53. Arunsan P, Ittiprasert W, Smout MJ, Cochran CJ, Mann VH, Chaiya$\operatorname{det} \mathrm{S}$, et al. Programmed knockout mutation of liver fluke granulin attenuates virulence of infection-induced hepatobiliary morbidity. Elife. 2019;8:e41463

54. Ittiprasert W, Mann VH, Karinshak SE, Coghlan A, Rinaldi G, Sankaranarayanan $\mathrm{G}$, et al. Programmed genome editing of the omega-1 ribonuclease of the blood fluke Schistosoma mansoni. Elife. 2019;8:e41337.

55. Brinkman EK, Kousholt AN, Harmsen T, Leemans C, Chen T, Jonkers J, et al. Easy quantification of template-directed CRISPR/Cas9 editing. Nucleic Acids Res. 2018:46:e58.

56. Brinkman EK, van Steensel B. Rapid quantitative evaluation of CRISPR genome editing by TIDE and TIDER. Methods Mol Biol. 2019;1961:29-44.

57. Hsiau T, Maures T, Waite K, Yang J, Kelso R, Holden K, et al. Inference of CRISPR edits from Sanger Trace Data. bioRxiv. 2018:251082.

58. Livak KJ, Schmittgen TD. Analysis of relative gene expression data using real-time quantitative PCR and the 2(-Delta Delta $C(T))$ method. Methods. 2001;25:402-8

59. Bayne CJ, Loker ES, Yui MA, Stephens JA. Immune-recognition of Schistosoma mansoni primary sporocysts may require specific receptors on Biomphalaria glabrata hemocytes. Parasite Immunol. 1984;6:519-28.

60. Lardans V, Boulo V, Duclermortier P, Serra E, Mialhe E, Capron A, et al. DNA transfer in a Biomphalaria glabrata embryonic cell line by DOTAP lipofection. Parasitol Res. 1996;82:574-6.

61. Symington LS, Gautier J. Double-strand break end resection and repair pathway choice. Annu Rev Genet. 2011;45:247-71.

62. Donovan KM, Leidinger MR, McQuillen LP, Goeken JA, Hogan CM, Harwani SC, et al. Allograft inflammatory factor 1 as an immunohistochemical marker for macrophages in multiple tissues and laboratoryanimal species. Comp Med. 2018;68:341-8.

63. De Zoysa M, Nikapitiya C, Kim Y, Oh C, Kang DH, Whang I, et al. Allograft inflammatory factor-1 in disk abalone (Haliotis discus discus): molecular cloning, transcriptional regulation against immune challenge and tissue injury. Fish Shellfish Immunol. 2010;29:319-26.

64. Li Q, Bai Z, Zhao L, Li J. Characterization of allograft inflammatory factor-1 in Hyriopsis cumingii and its expression in response to immune challenge and pearl sac formation. Fish Shellfish Immunol. 2016;59:241-9.

65. Liu G, Ma H, Jiang L, Zhao Y. Allograft inflammatory factor-1 and its immune regulation. Autoimmunity. 2007:40:95-102.

66. Roman E, Coman I, Prieto D, Alonso-Monge R, Pla J. Implementation of a CRISPR-based system for gene regulation in Candida albicans. mSphere. 2019;4:e00001-19.

67. van der Knaap WP, Loker ES. Immune mechanisms in trematode-snail interactions. Parasitol Today. 1990;6:175-82.

68. Vergote D, Bouchut A, Sautiere PE, Roger E, Galinier R, Rognon A, et al. Characterisation of proteins differentially present in the plasma of Biomphalaria glabrata susceptible or resistant to Echinostoma caproni. Int J Parasitol. 2005;35:215-24.

69. Negrao-Correa D, Mattos AC, Pereira CA, Martins-Souza RL, Coelho PM. Interaction of Schistosoma mansoni sporocysts and hemocytes of Biomphalaria. J Parasitol Res. 2012;2012:743920.

70. Guaraldo AM, Magalhaes LA, Rangel Hde A, Pareja G. Evolution of spopocysts of Schistosoma mansoni Sambon 1907 in Biomphalaria glabrata (Say 1818) and Biomphalaria tenagophila (d'Orbigny 1835). Rev Saude Publica. 1981;15:436-48.

71. Watano K, Iwabuchi K, Fujii S, Ishimori N, Mitsuhashi S, Ato M, et al. Allograft inflammatory factor-1 augments production of interleukin-6, -10 and -12 by a mouse macrophage line. Immunology. 2001;104:307-16.

72. Symeonidou I, Kourelis A, Frydas I, Karagouni E, Anogeianaki A, Hatzistilianou M, et al. Modulation of NF-kappa beta signalling pathways by parasites. J Biol Regul Homeost Agents. 2010;24:471-9.

73. Tato CM, Hunter CA. Host-pathogen interactions: subversion and utilization of the NF-kappa B pathway during infection. Infect Immun. 2002;70:3311-7.

74. Egana-Gorrono L, Chinnasamy P, Casimiro I, Almonte VM, Parikh D, Oliveira-Paula GH, et al. Allograft inflammatory factor-1 supports macrophage survival and efferocytosis and limits necrosis in atherosclerotic plaques. Atherosclerosis. 2019;289:184-94. 
75. Lin S, Staahl BT, Alla RK, Doudna JA. Enhanced homology-directed human genome engineering by controlled timing of CRISPR/Cas9 delivery. Elife. 2014;3:e04766.

76. Lino CA, Harper JC, Carney JP, Timlin JA. Delivering CRISPR: a review of the challenges and approaches. Drug Deliv. 2018;25:1234-57.

77. Fajrial AK, He QQ, Wirusanti NI, Slansky JE, Ding X. A review of emerging physical transfection methods for CRISPR/Cas9-mediated gene editing. Theranostics. 2020;10:5532-49.

78. Tsai SQ, Nguyen NT, Malagon-Lopez J, Topkar WV, Aryee MJ, Joung JK. CIRCLE-seq: a highly sensitive in vitro screen for genome-wide CRISPRCas9 nuclease off-targets. Nat Methods. 2017;14:607-14.
79. Huang J, You W, Xu Z, Yan Q, Shi C, Tang B, et al. An effective microinjection method and TALEN-mediated genome editing in Pacific abalone. Mar Biotechnol (NY). 2019;21:441-7.

\section{Publisher's Note}

Springer Nature remains neutral with regard to jurisdictional claims in published maps and institutional affiliations.
Ready to submit your research? Choose BMC and benefit from:

- fast, convenient online submission

- thorough peer review by experienced researchers in your field

- rapid publication on acceptance

- support for research data, including large and complex data types

- gold Open Access which fosters wider collaboration and increased citations

- maximum visibility for your research: over 100M website views per year

At BMC, research is always in progress.

Learn more biomedcentral.com/submissions 